\title{
Quantifying the breakdown of the Born-Oppenheimer approximation in surface chemistry
}

\author{
Igor Rahinov, ${ }^{* a}$ Russell Cooper, ${ }^{b c d}$ Daniel Matsiev, ${ }^{d e}$ Christof Bartels, ${ }^{b c}$ \\ Daniel J. Auerbach ${ }^{d}$ and Alec M. Wodtke ${ }^{b c d}$
}

Received 9th February 2011, Accepted 18th May 2011

DOI: $10.1039 / \mathrm{c1cp20356h}$

The Born-Oppenheimer Approximation (BOA) forms the basis for calculating electronically adiabatic potential energy surfaces, thus providing the framework for developing a molecular level understanding of a variety of important chemical problems. For surface chemistry at metal surfaces, it is now clear that for some processes electronically nonadiabatic effects can be important, even dominant; however, the magnitude of BOA breakdown may vary widely from one chemical system to another. In this paper we show that molecular-beam surface scattering experiments can be used to derive quantitative information about the magnitude of BOA breakdown. A state-to-state rate model is used to interpret the pre-exponential factor of the well-known Arrhenius surface temperature dependence of the electronically nonadiabatic vibrational excitation. We also show that reference to a "thermal limit" provides a quick and simple rule of thumb for quantifying BOA breakdown. We demonstrate this approach by comparing electronically nonadiabatic vibrational inelasticity for $\mathrm{NO}(\nu=0 \rightarrow 1)$ to $\mathrm{NO}\left(\nu=15 \rightarrow \nu^{\prime} \ll 15\right)$ and show that the electronically nonadiabatic coupling strengths are of a similar magnitude. We compare experiments for $\mathrm{NO}$ and $\mathrm{HCl}$ scattering from $\mathrm{Au}(111)$ and derive the quantitative relative magnitude for the electronically nonadiabatic influences in each system. The electronically nonadiabatic influences are 300-400 times larger for $\mathrm{NO}$ than for $\mathrm{HCl}$, for incidence energies near $0.9 \mathrm{eV}$.

${ }^{a}$ Department of Natural Sciences, The Open University of Israel, 108 Ravutski St., Raanana 43107, Israel.E-mail: igorra@openu.ac.il

${ }^{b}$ Institute of Physical Chemistry, Georg-August University of Göttingen, Göttingen, Germany

${ }^{c}$ Max Planck Institute for Biophysical Chemistry, Göttingen, Germany

${ }^{d}$ Department of Chemistry and Biochemistry,

University of California Santa Barbara, Santa Barbara, CA, USA

${ }^{e}$ SRI International, 333 Ravenswood Ave., Menlo Park, CA 94025, USA

\section{Introduction}

The typically large energy spacing between a molecule's electronic states, in comparison to its rotation and vibration states, reflects a vast time scale difference between electronic motion and the motion of nuclei. By fixing the nuclei at defined positions, the electronic structure calculation can be performed

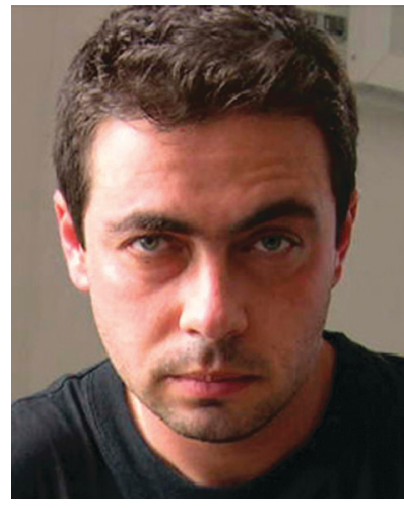

Igor Rahinov
Igor Rahinov is a lecturer at The Open University of Israel (OUI). He obtained his BSc in Chemistry from the Tel Aviv University in 2000, where he also earned his PhD in Chemical Physics under supervision of Professor Sergey Cheskis in 2006. He then joined the group of Professor Alec Wodtke at UC Santa Barbara as Fulbright postdoctoral fellow till 2009. After internship at the Weizmann Institute of Science (2009-2010) with Professor Daniel Zajfman he accepted his current lecturer position at OUI. His research focuses on development of novel methodologies for nanoparticles' production in reactive flows for catalytic and gas-surface scattering applications.

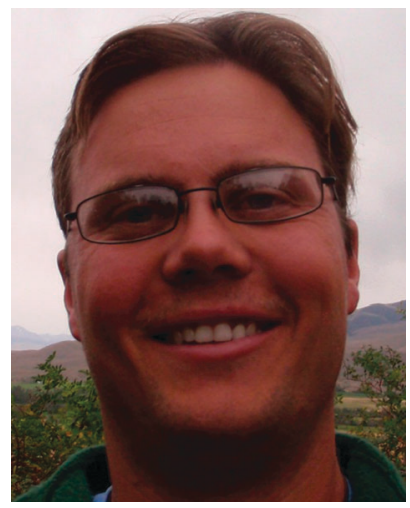

Russell Cooper
Russell Cooper obtained his BS degree from Montana State University in 2006 and during this time worked in the group of Tim Minton. He started his PhD the same year in University of California Santa Barbara in the group of Alec Wodtke. In 2008 he was awarded a PIRE-ECCI fellowship and spent half $a$ year in the group of Xueming Yang in Dalian, China. Russ and the Wodtke group moved to University Göttingen in Germany in 2010 where he will be receiving his doctoral degree. 
neglecting the coupling between nuclei and electrons: the Born-Oppenheimer approximation (BOA). ${ }^{14}$ This calculation may be carried out at a variety of nuclear positions revealing an effective potential energy surface upon which the nuclei move within the field of the electrons on the electronically adiabatic potential energy surface. It is difficult to overstate the importance of the BOA in chemistry. To quote a wellknown theoretical chemist: "It is not an exaggeration to say that the single activity that has demanded the most effort (both intellectual and computational) from theoretical chemists in the twentieth century is the electronic structure problem." 17 Furthermore, the BOA lies at the heart of the field of classical and quantum dynamics simulations, a profoundly important area of study relevant to a host of molecular phenomena: ranging from elementary chemical reactions, to biology, materials science, and heterogeneous catalysis.

It is therefore interesting to consider cases where the BOA fails; situations where the electronic motion can no longer adiabatically follow the nuclear motion involved in the chemistry. Molecular encounters with solid metal surfaces are one class of chemical phenomena exhibiting electronically nonadiabatic behavior. Metallic solids exhibit a continuum of electronic states. This means that a conduction band electron can be promoted above the Fermi level leaving a hole behind below the Fermi level and this excited electron-hole pair (EHP) may have an arbitrarily large or small energy. Hence, the EHP excitation spectrum always possesses a resonance with the ro-vibrational quantum transitions of a molecule. To the extent that a coupling between the metal's electrons and the heavy atom motion of the molecule exists, the BOA will break down.

Despite the now indisputable experimental evidence demonstrating the existence of electronically nonadiabatic influences in molecule-surface interactions-some of which will be presented in this article - there is still no scientific consensus concerning their general importance. This is partly due to the

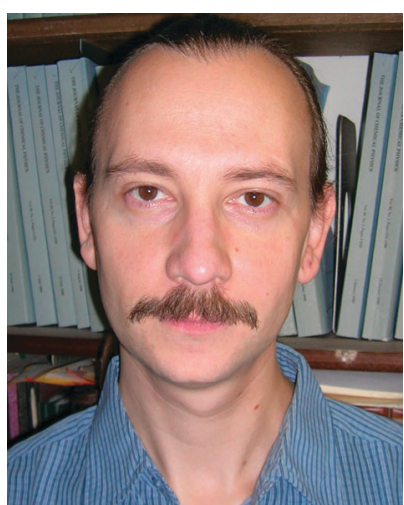

Daniel Matsiev
Daniel Matsiev was born in Soviet Union in 1973. He received his $B S$ and $M S$ degrees in physics and applied mathematics from Moscow Institute of Physics and Technology in 1994 and 1996. After relocating to USA in 1997 he played a key role in several research projects at University of California, Santa Barbara. He received his PhD from UCSB in 2007 with Professor Alec Wodtke, studying electronically nonadiabatic vibrational energy transfer in molecular collisions with metal surfaces. Currently he is a research scientist at SRI International working on collisional energy transfer and spectroscopy of molecules important in planetary atmospheric studies.

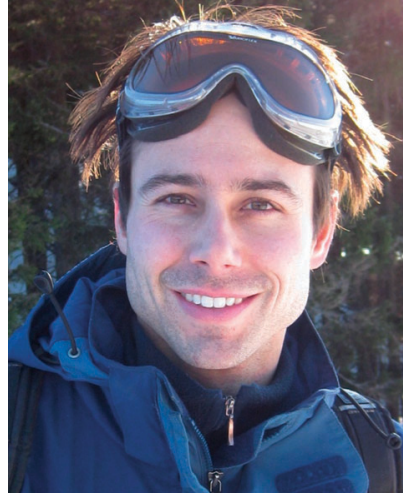

Christof Bartels
Christof Bartels received his PhD in 2008 from the University of Freiburg, where he worked on angle resolved photoelectron spectroscopy on size-selected clusters under the supervision of Professor Bernd von Issendorff. After postdoc positions with Professor Akira Terasaki in Tokyo and with Professor Alec Wodtke in Santa Barbara, he is now at the University of Götingen. His current research concentrates on nonadiabatic effects in the conversion of energy in molecule-surface collisions.

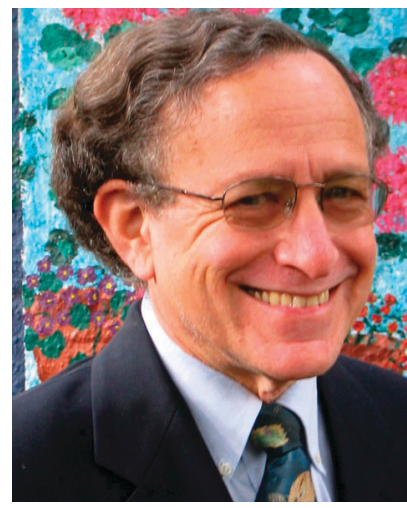

Daniel J. Auerbach
Daniel Auerbach is perhaps best known as a pioneer in the application of molecularbeam and laser-spectroscopic techniques to understanding of the chemical dynamics at surfaces. Auerbach is currently an adjunct professor in the Department of Chemistry and Biochemistry at University of California in Santa Barbara. Previously he was CTO of GRT Inc., served for 10 years as Department Group Manager of the Science and Technology Department at the IBM Almaden Research Center. Auerbach holds a PhD degree in Physics from the University of Chicago.

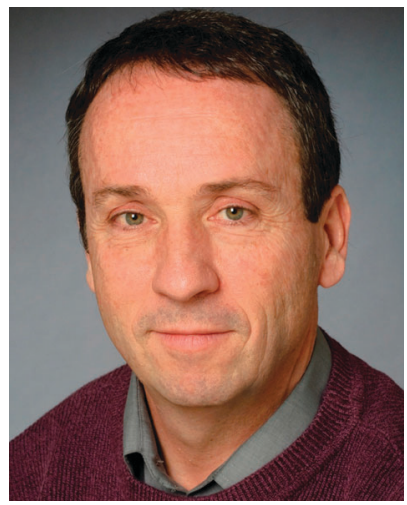

Alec M. Wodtke
Since 2010 Alec Wodtke has been an Alexander-von-HumboldtProfessor at the Georg-August Universität Göttingen and the director of the Dynamics at Surfaces Department at the Max Planck Institute for Biophysical Chemistry. After graduating from the University of Utah in 1981, he did his PhD at UC Berkeley until 1986. He then post-doc'd at the MPI for Fluid Dynamics in Göttingen until 1988. Thereafter, he became an Asst. Prof. and eventually a full professor at UC Santa Barbara in 1996. His experiments concern novel pathways of energy conversion in chemical reactions at surfaces and have stimulated new theories of surface chemistry. 
overwhelming popularity and success of density functional theory (DFT) in calculating electronically adiabatic interaction potentials for molecules at metal surfaces. A perhaps more important point is the lack of detailed and rigorous efforts to quantify the magnitude of electronically nonadiabatic influences in molecule surface interactions. Too often qualitative language - "strong, weak, large, small"- takes the place of quantification of electronically nonadiabatic effects.

In this perspective article we first review some of the most dramatic experimental examples of electronically nonadiabatic behavior of molecules at metal surfaces. We then go on to present a new approach to understanding inelastic moleculesurface scattering data that provides a quantitative description of the magnitude of the nonadiabatic effects. We show how this allows one to compare the importance of electronically nonadiabatic influences between different molecules interacting with different surfaces. Finally, we suggest future directions for this line of research.

\section{Key experiments demonstrating electronically nonadiabatic behavior}

Molecular beam scattering experiments have long played an important role in unraveling the energy-transfer dynamics at the gas-surface interface. ${ }^{18-21}$ When combined with laser-based state-specific preparation and detection, molecular beam scattering techniques allow for accurate control of the initial translational energy, internal degrees of freedom, and incidence angle of the scattering molecules. ${ }^{22-24}$ The surface temperature, $T_{\mathrm{S}}$, and incidence energy, $E_{\mathrm{I}}$, dependence of $\nu=0 \rightarrow 1$ vibrational excitation occurring in $\mathrm{NO}$ collisions with $\mathrm{Ag}(111)$ revealed some of the dynamical signatures of electronically nonadiabatic interactions. ${ }^{6,25}$ Specifically two such signatures were seen as shown in Fig. 1(a and b): (1) an Arrhenius $T_{\mathrm{S}}$ dependence exhibiting an effective activation energy equal to the vibrational excitation energy and (2) a threshold free but strong dependence of the vibrational excitation probability on $E_{\mathrm{I}}$. Qualitatively different dynamical signatures for electronically adiabatic vibrational energy transfer observed for a different system $\left(\mathrm{NH}_{3} / \mathrm{Au}(111)\right)-E_{\mathrm{I}}$ dependence with vibrational thresholds and weak or absent $T_{\mathrm{S}}$ dependence - are shown for comparison in Fig. 1(c and d). ${ }^{15,26}$

Fig. 1a shows the $T_{\mathrm{S}}$ dependence typical in such experiments, which is described by a formula characterizing the population of excited EHPs in energy resonance with the NO vibrational transition (EHP-V coupling):

$$
P=A \exp \left(-\frac{\Delta E_{\mathrm{vib}}}{k_{\mathrm{b}} T_{\mathrm{S}}}\right)
$$

While the Arrhenius form with an activation energy equal to $\Delta E_{\mathrm{vib}}$ is a qualitative signature for the role of nonadiabatic electronic transitions, the pre-exponent, $A$, contains information on the magnitude of the electronically non-adiabatic coupling, which has only recently been rigorously analyzed. ${ }^{4,12} \mathrm{We}$ return to this point in Section 3. The incidence energy dependence shown in Fig. $1 \mathrm{~b}$ is strong but, in contrast to the adiabatic case (Fig. 1d), exhibits no threshold. This proves that electronically nonadiabatic vibrational excitation is not a conversion of energy from incidence translation to vibration. Rather the
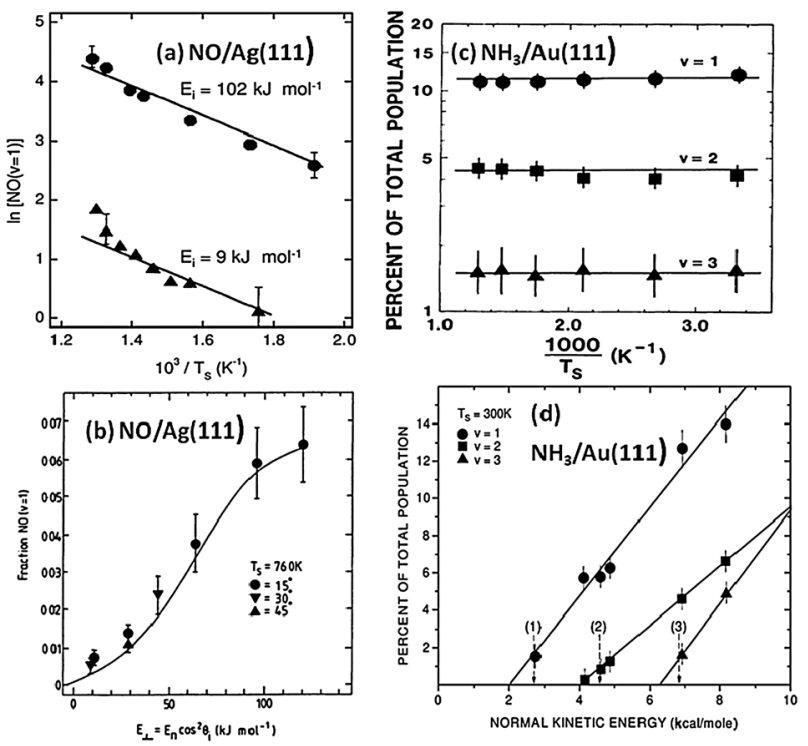

Fig. 1 Surface temperature, $T_{\mathrm{S}}$, and incidence energy, $E_{\mathrm{I}}$ dependence of vibrational excitation in molecule-surface collisions: adiabatic $v s$. nonadiabatic behavior. (a) Nonadiabatic: an Arrhenius dependence on $T_{\mathrm{S}}$ with an activation energy equal to the vibrational excitation energy is seen. See ref. 6. (b) Nonadiabatic: a strong dependence on $E_{\mathrm{I}}$ with an apparent threshold at $E_{\mathrm{I}}=0$. See ref. 10. (c) Adiabatic: $T_{\mathrm{S}}$ independent excitation probability. This example shows $\mathrm{NH}_{3}\left(v_{\text {umbrella }}=0 \rightarrow 1,2,3\right)$ on $\mathrm{Au}(111)$ at an incidence energy of $\sim 0.3 \mathrm{eV}$. See ref. 15. (d) Adiabatic: thresholds close to the vibrational excitation energies indicate conversion of incidence translational energy to molecular vibration. See ref. 15.

energy comes from the surface and specifically from the decay of thermally excited EHPs while the incidence energy enhances the coupling between EHPs and NO vibration..$^{2,6,10}$ Similar results are seen for $\mathrm{NO}$ scattering from $\mathrm{Cu}(110) .{ }^{16}$

EHP-V coupling can also be inferred from IR linewidth and lineshape measurements. ${ }^{27-32}$ For example, the IR linewidth of the stretch vibration of $\mathrm{CO}$ adsorbed on $\mathrm{Ni}(111)^{29}$ is greater than $\sim 5 \mathrm{~cm}^{-1}$ even at $T_{\mathrm{S}}=10 \mathrm{~K}$, where vibrational dephasing is unimportant. The linewidth is attributed to $\mathrm{CO}$ vibrational relaxation to the metal on the ps time scale. Asymmetric Fano lineshapes - see Fig. 2-were observed for W-H vibrational transitions in the IR for hydrogen covered W surfaces ${ }^{1}$ and shown to be a spectroscopic signature of strong EHP-V coupling. ${ }^{8}$ Infrared emission has been used to investigate low frequency modes. ${ }^{33-35}$ Here, it is unclear if the linewidths reflect electronically nonadiabatic coupling or enhanced coupling to phonons, which is expected at low frequency, ${ }^{34-36}$ or to inhomogeneous line broadening.

While the infrared linewidth approach to the analysis of energy transfer at surfaces should not be underestimated, uncertainties related to dephasing and sample inhomogeneity must (and often cannot) be overcome. ps and fs laser methods can be applied to these problems and confirm the interpretation of IR linewidth measurements. ${ }^{3,37,38}$ For example, vibrational relaxation of IR pumped $\mathrm{CO}$ adsorbates can be monitored by sum frequency generation $(\mathrm{SFG})^{3}$ and in other work by transient IR absorption spectroscopy. ${ }^{39}$ Fig. 3a shows an example of the former type of experiment for the $\mathrm{C}-\mathrm{O}$ stretch relaxation on $\mathrm{Cu}(100)$. The vibrational lifetime obtained here 


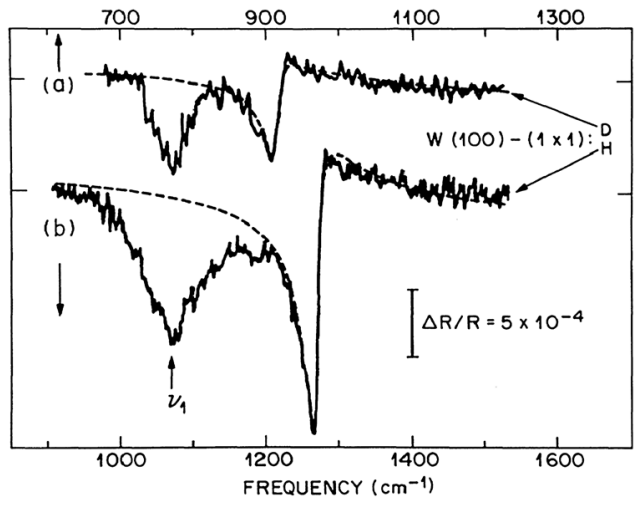

Fig. 2 Fano lineshapes in IR spectra of surface adsorbates. Vibrational spectra of D and $\mathrm{H}$ measured on H/D-saturated W(100) surface. ${ }^{1}$ The $v_{1}$ denotes the symmetric W-H(D) stretch. The higher frequency feature is the W-H(D) bend overtone. It exhibits a Fano lineshape characteristic of strong vibration to electron-hole pair (EHP-V) coupling. ${ }^{8}$ Adapted from ref. 1.

is also on the ps timescale. Vibrational lifetimes of adsorbates on insulators, where EHP-V coupling is unlikely, were reported for $\mathrm{CO}$ on $\mathrm{NaCl}(100) .{ }^{9}$ See Fig. 3b. Here the lifetime is on the ms time scale, a nine order of magnitude difference in comparison to $\mathrm{CO}$ on a metal. The Debye frequency of $\mathrm{NaCl}$ $\left(223 \mathrm{~cm}^{-1}\right)$ is so far out of resonance with the $\mathrm{CO}$ vibration $\left(2100 \mathrm{~cm}^{-1}\right)$ that only high-order multiphonon transitions are possible. Indeed coupling of $\mathrm{CO}$ vibration to the solid is so inefficient that intra-adsorbate energy pooling dominates the vibrational dynamics. ${ }^{40,41}$

Multi-quantum vibrational relaxation is seen when $\mathrm{NO}(\nu=15)$ collides with a metal surface; whereas, on an insulating $\mathrm{LiF}$ surface, vibrational relaxation is nearly absent. ${ }^{5,42,43}$ See Fig. 4. Here molecular beams of NO are prepared in high vibrational states by laser optical pumping and scattered molecules are probed by state selective laser based methods. ${ }^{13,18,19,44}$ The final vibrational state distribution shows that the most probable inelastic process is loss of $\sim 8$ vibrational quanta representing $\sim 1.6 \mathrm{eV}$. It was shown that it occurs in a direct scattering event on a sub-ps timescale, with little conversion of vibrational energy to NO rotation or translation, meaning the solid metal substrate is the dominant energy acceptor.

"Vibrational auto-detachment" is a possible mechanism explaining the efficient relaxation of highly vibrationally excited
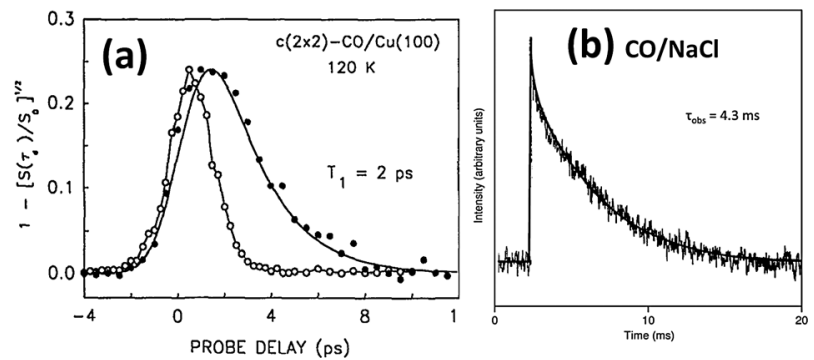

Fig. 3 Real time observations of vibrational lifetimes: nine orders of magnitude difference. (a) $\mathrm{CO}(\nu=1)$ relaxes on $\mathrm{Cu}(100)$ within a few ps. See ref. 3. (b) $\mathrm{CO}$ on $\mathrm{NaCl}$ relaxes in ms. See ref. 9. The electronic degrees of freedom in the metal efficiently accept vibrational energy from the $\mathrm{CO}$ molecule.

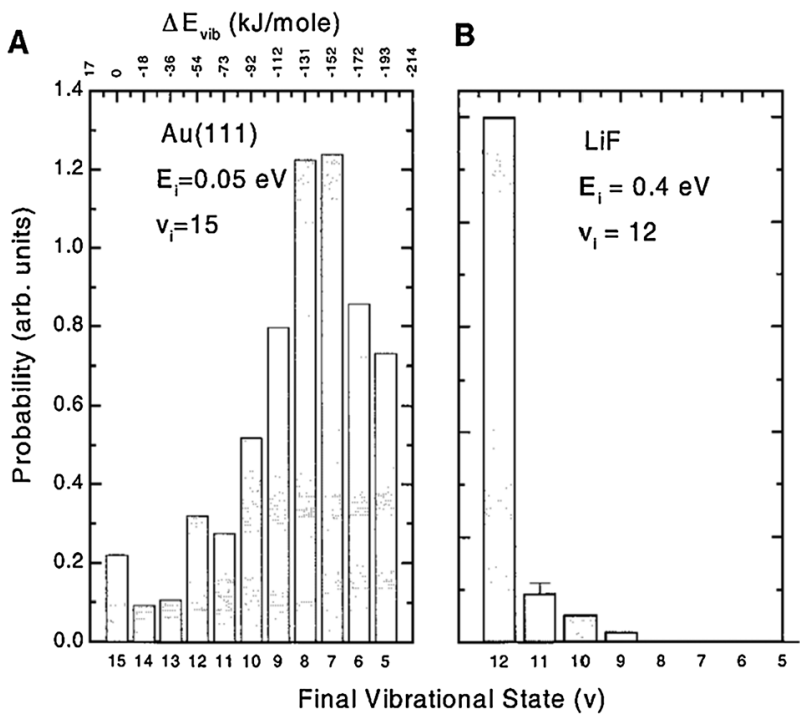

Fig. 4 Multi-quantum vibrational relaxation. (a) Vibrational distribution of scattered NO resulting from collisions of $\mathrm{NO}(\nu=15)$. (b) Similar measurements for NO on LiF. The electronic degrees of freedom of the metal can accept many vibrational quanta of vibration from the molecule. Adapted from ref. 5.

NO molecules on $\mathrm{Au}(111) .^{5}$ When the vibration of the NO molecule is near its outer classical turning point an electron at the Fermi energy may hop from the solid to the molecule, forming a transient $\mathrm{NO}^{-}$stabilized by its image charge. The $\mathrm{NO}^{-}$bond compresses within 25 fs to the inner classical turning point, raising the electron potential more than $2 \mathrm{eV}$ above the Fermi energy. Subsequent loss of the electron back to the solid produces NO in lower vibrational states and electronic excitation of the solid. ${ }^{43}$

Electronic friction based theories are capable of describing multi-quantum vibrational relaxation, ${ }^{45}$ but assume sequential single quantum EHP-V energy transfer processes. Experiments that probe the emission of electrons to the gas phase, where a large fraction of the vibrational energy must be transferred to a single electron, help substantiate the vibrational autodetachment mechanism. ${ }^{7,43,44,46,47}$ In these experiments sub-monolayer coverage of caesium on $\mathrm{Au}(111)$ lowered the work function to $1.6 \pm 0.1 \mathrm{eV} .^{7}$ When the vibrational energy of the NO exceeds the work function, electron emission is observed. See Fig. 5. The observed quantum yield was as high as $\sim 10 \%$. These results strongly suggest that a large fraction of the vibrational energy lost from the molecule appears in the excitation energy of a single electron.

Direct measurements of the ejected electron's kinetic energy are shown in Fig. 6, where $\operatorname{NO}(\nu=16)$ has been scattered from $\mathrm{Cs} / \mathrm{Au} .{ }^{11}$ The most probable exo-electron kinetic energy is $0.5 \mathrm{eV}$ with respect to the vacuum level (lower $x$-axis) and $2.1 \mathrm{eV}$ with respect to the Fermi level (upper $x$-axis). This is $64 \%$ of the incidence vibrational energy $-3.3 \mathrm{eV}$ - and corresponds to vibrational relaxation from $\mathrm{NO}(\nu=16)$ to at least as low as $\operatorname{NO}(\nu=5)$. The maximum observed electron kinetic energy, shown in Fig. 6 as $E_{\mathrm{MAx}}^{\mathrm{T}}$, represents 0.95 of the incidence vibrational energy. Interestingly, the observed exoelectron kinetic energy distribution is broad and does not 


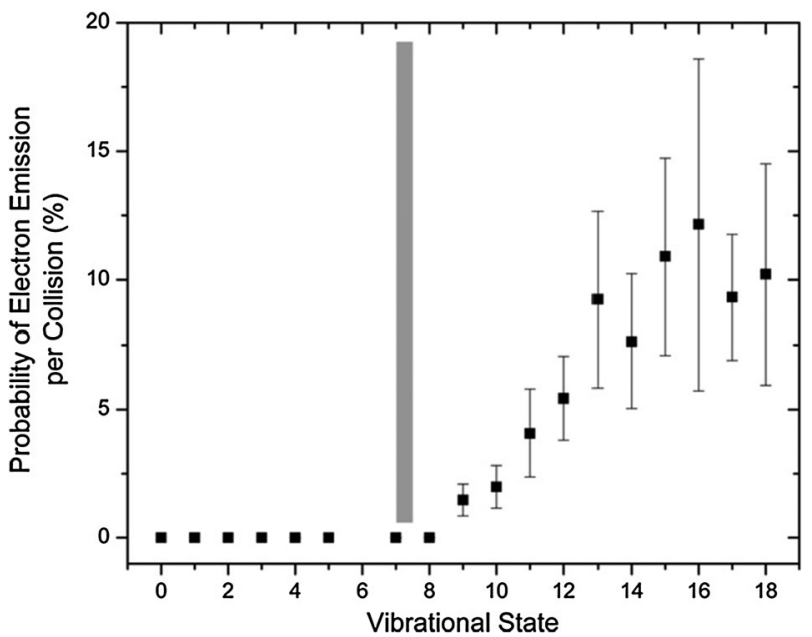

Fig. 5 Absolute quantum yield of vibrationally promoted electron emission measured vs. vibrational quantum state, $v$, of the $\mathrm{NO}(v)$ molecule impinging the low-work function caesium-decorated gold surface. The grey bar denotes the measured value of the surface work function. ${ }^{7}$ Adapted from ref. 13 and ref. 46.

exhibit any substructure that might be expected from the quantized vibrational states of NO molecules. While this may reflect the nature of the vibrational auto-detachment mechanism, it is important to note that the instrumental resolution of $0.15 \mathrm{eV}$ is insufficient to resolve the NO vibrational spacing of $\sim 0.2 \mathrm{eV}$.

In contrast to previously described and documented mechanisms of exoelectron emission, ${ }^{48-53}$ the probability of vibrationally promoted electron emission decreases with increased incidence translational energy. ${ }^{47}$ Indeed the electron emission velocity dependence is well characterized by an inverse, $1 / v$, relation, shown in Fig. 7.

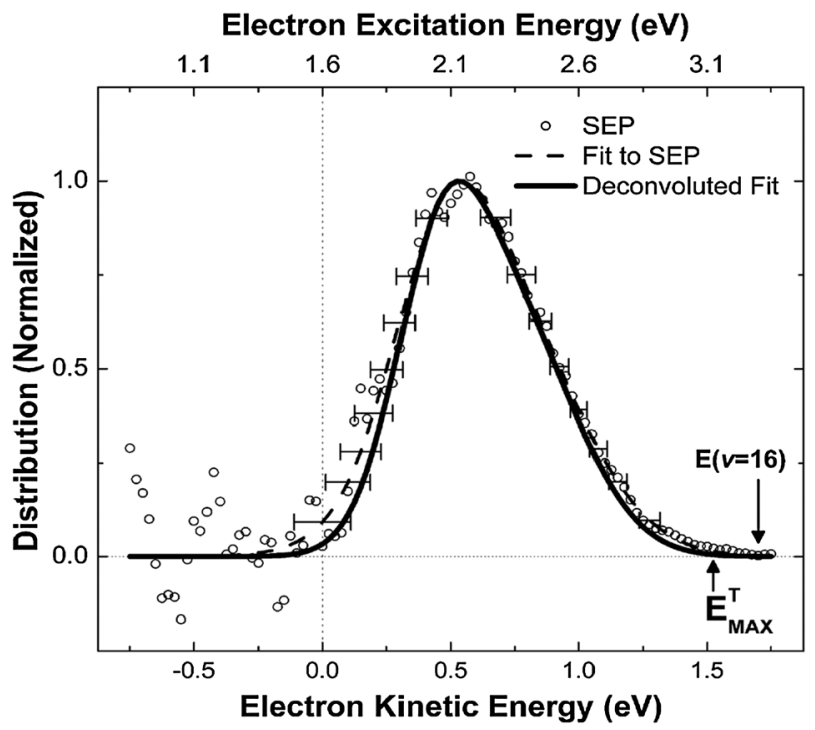

Fig. 6 Electron kinetic energy distribution resulting from vibrationally promoted- $\mathrm{NO}\left(\nu=16, E_{\mathrm{vib}}=3.3 \mathrm{eV}\right)$ - electron emission from Cs $/ \mathrm{Au}$. The upper $x$-axis is the electron energy with respect to the Fermi level, adding the work function $\varphi=1.6 \pm 0.1 \mathrm{eV}^{7}$ to the observed electron kinetic energy. See ref. 11.

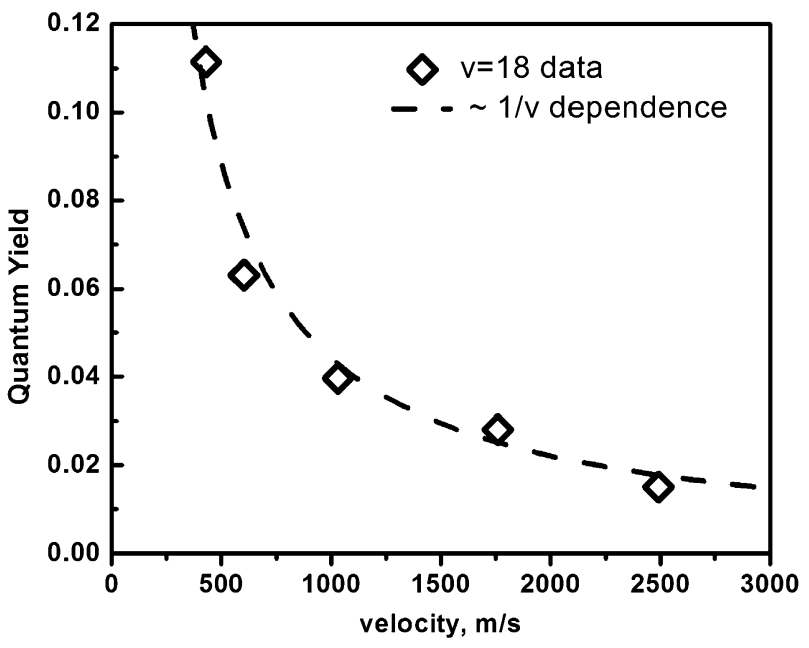

Fig. 7 Inverse velocity dependence for vibrationally promoted electron emission. Here, $\mathrm{NO}(v=18)$ collides with a $\mathrm{Cs} / \mathrm{Au}$ surface and ejected electrons are detected. This observation is a sign that the electron emission process is approximately independent of translation energy and depends only on the time spent in a critical region near the surface. See text.

The $1 / v$ dependence may arise as follows. Electron transfer to the NO molecule is impossible until a certain critical outer distance, $z_{>}$, is reached. Furthermore, electron escape from the NO anion is impossible if the anion is closer than a critical inner distance $z_{<}$. At distances less than $z_{<}$the image charge stabilization plus the surface work function exceeds the initial vibrational energy. Reasonable estimates of $z_{>}$and $z_{<}$suggest that a constant velocity approximation is valid for $z_{>}>z>z_{<}$. That is the NO interaction potential is flat in this region. If the probability for vibrationally promoted electron emission were itself velocity independent, then only the time spent between $z_{>}$and $z_{<}$would matter, and a kind of window of opportunity would describe the dynamics. This leads to an inverse velocity dependence.

\section{A quantitative approach to vibrational overtone excitation of NO}

In the previous section of this article we described selected experimental observations that leave little doubt as to the existence of electronically nonadiabatic interactions in molecule surface encounters. Of course, numerous examples in surface chemistry exhibit little evidence of electronically nonadiabatic behavior. Establishing a quantitative criterion or means of analysis that would allow the comparison of one molecule/ surface system to another would improve our ability to evaluate the importance of electronically nonadiabatic interactions in general. In this section we describe experiments suggesting that a quantitative analysis of the magnitude of the electronically nonadiabatic interactions is possible.

One concrete question that we will address is the relationship between the behavior of NO in high vibrational states where multiquantum vibrational relaxation is seen (see Section 2 and the discussion surrounding Fig. 4) and the behavior of NO in low vibrational states, where Arrhenius $T_{\mathrm{s}}$-dependence and threshold-less $E_{\mathrm{I}}$-dependence are seen. See Section 2 and 
the discussion surrounding Fig. 1. One might reasonably ask whether the several $\mathrm{eV}$ difference in incidence energy of vibration is not sufficient to elicit qualitatively different dynamics. For multiquantum vibrational relaxation of highly vibrationally excited NO large vibrational energy jumps, $\Delta \nu \approx 8$, lead to large energy excitations of individual electrons; even electron emission can be seen. Is the behavior in low vibrational states comparable or does it result from an essentially different set of circumstances?

As is shown in Fig. 1 and 4, for low vibrational states, the most probable event is vibrationally elastic scattering, whereas for high vibrational states the most dominant change in vibrational states is $\sim 8$ quanta. From this, one might simply conclude that the electronically nonadiabatic influences are much stronger for high vibrational states than for low. We will show that this simple conclusion is unjustified. The behavior of NO scattering from $\mathrm{Au}(111)$ when it is in its ground vibrational state is not only qualitatively similar to scattering in high vibrational states, but according to the analysis we will present the electronically nonadiabatic coupling is of a similar magnitude. More specifically, we will show how the electronically nonadiabatic coupling strength may be gauged in relationship to a thermal limit. This allows a quantitative experimental criterion to be established describing the strength of electronically nonadiabatic coupling.

We first consider vibrational excitation experiments showing $\mathrm{NO}(\nu=0)$ scattering from $\mathrm{Au}(111)$ results in both single quantum $(\nu=0 \rightarrow 1)$ and multi-quantum $(\nu=0 \rightarrow 2)$ excitation. ${ }^{4,12}$ Through a quantitative analysis one may show that for multi-quantum excitation, vibrational overtone excitation is dominant. That is, one direct $0 \rightarrow 2$ transition prevails over two sequential single-quantum transitions, a process that is analogous to multiquantum vibrational relaxation of highly vibrationally excited NO.

To compare electronically nonadiabatic vibrational excitation to de-excitation, we must devise a means of analysis that allows us to look beyond thermal factors. That is to say, every electronically nonadiabatic energy transfer process will have a "thermal limit". For vibrational de-excitation, vibrational energy excites EHPs. For vibrational excitation, one requires thermally excited EHPs to relax transferring energy to NO vibration. Thus for a finite $T_{\mathrm{S}}$, vibrational de-excitation is always more efficient than excitation. Consider the case where the EHP-V coupling is so strong that the vibrational relaxation of $\mathrm{NO}$ from $\nu=1$ to $\nu=0$ reaches the thermal limit in a collision with a room temperature surface. In this case the final $\nu=1$ population will be $\mathrm{O}\left(10^{-4}\right)$ that of $\nu=0$. The same thermal limit in vibrational excitation of $\nu=0$ produces a $\nu=1$ population to be $\mathrm{O}\left(10^{-4}\right)$. In the former case, one observes near complete relaxation, whereas in the latter it will be $\mathrm{O}\left(10^{-4}\right)$ excitation efficiency. If we look only at the probability of vibrational energy transfer we might conclude incorrectly that vibrational relaxation exhibits much stronger nonadiabatic effects than vibrational excitation. By looking at these events in relation to the thermal limit, we see that both are examples of strong nonadiabatic effects.

A similar logic applies to the analysis of vibrational overtone excitation. The energy of EHPs driving $\Delta \nu=+2$ transitions must be twice as high as that of EHPs driving $\Delta \nu=+1$ transitions. Thus, for a given surface temperature the probability of surface collision induced overtone vibrational excitation will always be significantly smaller than that of the fundamental $(\Delta \nu=1)$ due to the limited thermal population of high energy EHPs. Only when one accounts for these thermal factors, the intrinsic strength of the electronically nonadiabatic coupling is obtained. This is best shown by considering a specific experiment.

Fig. 8 shows REMPI spectra of $\mathrm{NO}(\nu=1,2)$ populated by collisions of $\mathrm{NO}(\nu=0)$ on $\mathrm{Au}(111)$ with $E_{\mathrm{I}}=0.93 \mathrm{eV}$. The $\nu=2$ signal is more than an order of magnitude weaker than that of $\nu=1$ and the dependence of the REMPI signal on $T_{\mathrm{S}}$ is stronger for $\nu=2$ than for $\nu=1$. This is consistent with an Arrhenius $T_{\mathrm{S}}$ dependence expected for electronically nonadiabatic vibrational excitation, eqn (1). In the Arrhenius expression, the pre-factor, $A$, is related to the intrinsic coupling between EHPs and molecular vibration, while the exponential term represents the statistical likelihood to find a thermally excited EHP of the correct energy to excite molecular vibration.

Fig. 9 shows absolute excitation probabilities for the two vibrational channels and their dependence on $T_{\mathrm{S}}{ }^{4}$ The solid lines are the results of a kinetic model that we will describe shortly. The dash-dot lines, indistinguishable from the solid lines, are best fit Arrhenius functions with the activation energies set equal to the $\Delta E_{\mathrm{vib}}$ for the two channels. The thermal limits are also shown for comparison as a dotted line. One can see that the vibrational excitation probabilities are about half the thermal limit. This simple comparison already serves as a semi-quantitative estimate of the strength of the electronically nonadiabatic coupling.

One may take this idea still further. From the Arrhenius $T_{\mathrm{S}}$ dependence one cannot distinguish sequential two-quantum from direct overtone excitation. Assuming that the fundamental collision-induced vibration excitation follows an Arrhenius dependence with an activation energy equal to one vibration quantum of energy, the probability to excite $\nu=2$ in two

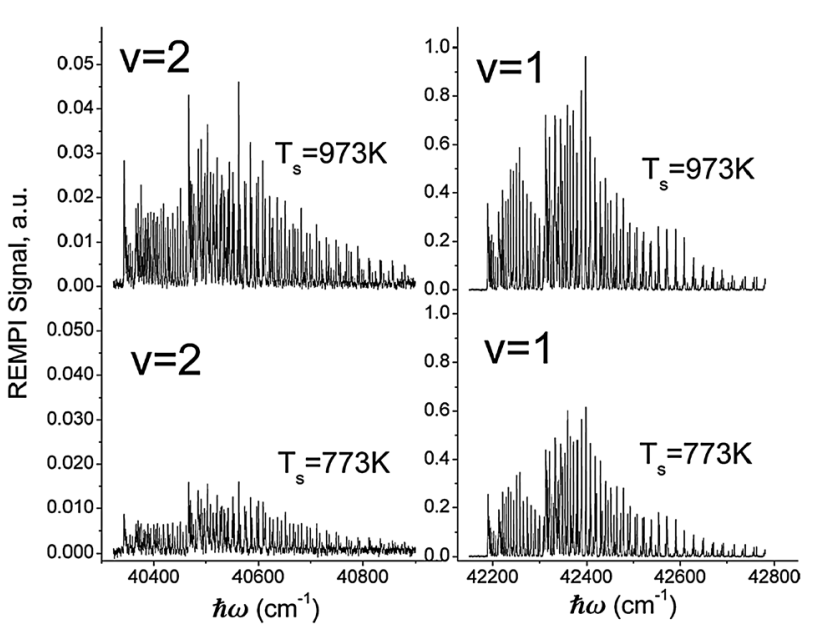

Fig. 8 Typical rotationally-resolved REMPI spectra of $\operatorname{NO}(\nu=1,2)$ observed when $\mathrm{NO}(\nu=0)$ is scattered from $\mathrm{Au}(111)$ at $E_{\mathrm{I}} \approx 0.9 \mathrm{eV}$ and $T_{\mathrm{S}}$ of 773 and $973 \mathrm{~K}$. Adapted from ref. 4. 


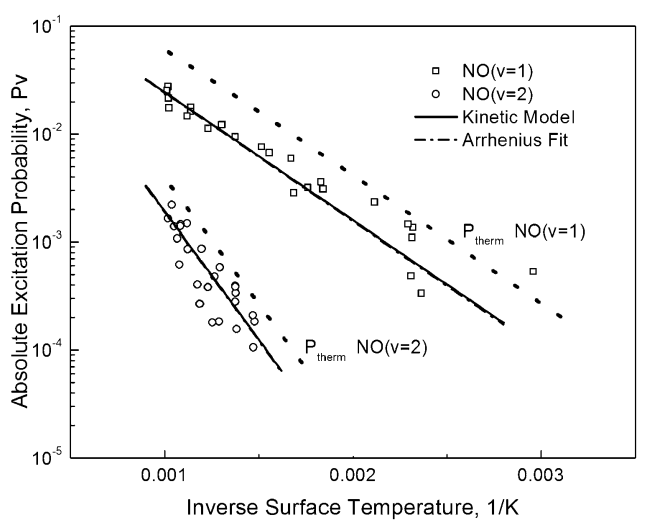

Fig. $9 T_{\mathrm{S}}$ dependence of the vibrational excitation probabilities for $\mathrm{NO}(\nu=0 \rightarrow 1)$ and $\mathrm{NO}(\nu=0 \rightarrow 2)$. Open symbols denote experimental data. Dotted lines denote the thermal limits for both excitation channels. Solid lines result from a kinetic model described in the text. Dash-dot lines (indistinguishable from the solid lines) show Arrhenius fits ${ }^{4}$ with $E_{\mathrm{a}, 0-1}=0.236 \mathrm{eV}, A_{\mathrm{exp}, 1}=0.38$ and $E_{\mathrm{a}, 0-2}=0.472 \mathrm{eV}, A_{\mathrm{exp}, 2}=0.46$. See ref. 12 .

independent steps also results in an Arrhenius form with twice the activation energy.

$$
\begin{aligned}
P_{\text {direct }}(0-2) & =A_{02} \exp \left(-\frac{2 h \nu}{k_{\mathrm{B}} T_{\mathrm{S}}}\right) \\
P_{\text {sequential }}(0-1-2) & =P_{\text {direct }}(0-1) P_{\text {direct }}(1-2) \\
& =A_{01} \exp \left(-\frac{h \nu}{k_{\mathrm{B}} T_{\mathrm{S}}}\right) A_{12} \exp \left(-\frac{h \nu}{k_{\mathrm{B}} T_{\mathrm{S}}}\right) \\
& =A_{\mathbf{0 1}} A_{\mathbf{1 2}} \exp \left(-\frac{2 h \nu}{k_{\mathrm{B}} T_{\mathrm{S}}}\right)
\end{aligned}
$$

However, one may use the experimentally derived pre-exponential factors to distinguish these two cases. The experimentally derived absolute excitation probabilities shown in Fig. 9 lead to physically interesting pre-exponential factors for each channel, $A_{\text {exp }, 1}=0.38$ and $A_{\text {exp }, 2}=0.46$ (where $A_{\text {exp }, 1}$ and $A_{\text {exp }, 2}$ are defined in the caption of Fig. 9). The pre-exponential factor may be approximately thought of as a $T \rightarrow \infty$ limit; that is, where thermal populations of EHPs are sufficient to promote the molecule to any vibration level. Moreover, all pre-exponential factors are bounded from above by 1 and from below by 0 : $0 \leq A \leq 1$. The fact that $A_{\text {exp }, 2}>A_{\text {exp }, 1}$ immediately rules out a dominant sequential single-quantum mechanism. In this case $A_{\text {exp }, 1}=A_{01}$ and $A_{\text {exp }, 2}=A_{01} A_{12}$ and, as it is impossible to multiply two numbers between 0 and 1 and get a larger number, this mechanism can be excluded from playing a major role in the vibrational excitation.

Let us think more deeply about the thermal limit. In electronically nonadiabatic vibrational excitation of ground vibrational state molecules, the highest probability that can be reached is if the molecule comes into thermal equilibrium with the EHP bath in the solid, which is held at $T_{\mathrm{S}}$. In this case the probability to find a molecule in the vibrational state $\nu$ is simply given by the Boltzmann factor at the temperature $T_{\mathrm{S}}{ }^{4,12}$ that is:

$$
P_{\text {therm }}=\exp \left(-\frac{\Delta E_{\mathrm{vib}}}{k_{\mathrm{B}} T_{\mathrm{S}}}\right) / Q_{\mathrm{vib}}\left(T_{\mathrm{S}}\right)
$$

where $\Delta E_{\mathrm{vib}}$ is the molecule's vibration spacing and $Q_{\mathrm{vib}}\left(T_{\mathrm{S}}\right)$ is the partition function of harmonic oscillator given by $\left[1-\exp \left(-h \nu / k_{\mathrm{B}} T_{\mathrm{S}}\right)\right]^{-1}$.

It is then clear that the Arrhenius expression does not describe the transition to this strong coupling limit, since eqn (4) is not of an Arrhenius form. To address this issue, a simple rate model was developed ${ }^{4,12}$ for vibrational state changing events occurring in collisions with a metal surface that incorporates microscopic reversibility and a statistical treatment of the thermal energy distribution of metallic EHPs. The details of this model and its implications exemplified on the NO/Au(111) excitation are elaborated next.

Persson and Persson ${ }^{54}$ used a Fermi Golden Rule approach to characterize the electronically nonadiabatic vibrational relaxation of $\mathrm{CO}$ on $\mathrm{Cu}(100)$, where the rate of EHP-V energy transfer is given by:

$$
k_{\nu \nu^{\prime}}=\frac{2 \pi}{\hbar} \sum_{i, k}\left|\left\langle i \nu\left|H^{\prime}\right| k \nu^{\prime}\right\rangle\right|^{2} \delta\left(\varepsilon_{i}-\varepsilon_{k}-\hbar \omega_{0}\right)
$$

where $\nu$ and $\nu^{\prime}$ are initial and final vibrational states of the molecule, $\varepsilon_{i}$ and $\varepsilon_{k}$ are energies of the initial $|i\rangle$ and final $|k\rangle$ electronic states in the metal, $\omega_{0}$ is the vibrational frequency, and $H^{\prime}$ is the perturbation corresponding to the AndersonNewns electronic Hamiltonian. ${ }^{55,56}$ Assuming that such a perturbation theory-based approach can be generally validnot only for single quantum but also for direct multi-quantum vibrational energy transfer, and taking into account the finite temperature of the surface by incorporating the Fermi-Dirac distribution function for the electrons in the conduction band of the metal the expression for the rate of vibrational energy transfer was derived: ${ }^{12}$

$$
k_{\nu \nu^{\prime}}=a_{\nu \nu^{\prime}} \frac{E_{\nu \nu^{\prime}}}{\exp \left(-\frac{E_{\nu \nu^{\prime}}}{k_{\mathrm{b}} T_{\mathrm{S}}}\right)-1} \quad \text { with } \quad a_{\nu \nu^{\prime}}=\frac{2 \pi}{\hbar} \lambda_{\nu \nu^{\prime}}^{2} \rho^{2}
$$

where $E_{\nu \nu^{\prime}}$ is the vibrational energy change, $\lambda_{v v}^{\prime}=\left|\left\langle i \nu\left|H^{\prime}\right| k \nu^{\prime}\right\rangle\right|$ is the matrix element coupling the electronic and vibrational degrees of freedom, $\rho$ is the density of states projected onto the molecular affinity level, $k_{\mathrm{b}}$ is the Boltzmann constant, and $T_{\mathrm{S}}$ is the surface temperature.

The model ${ }^{4,12}$ further assumes that the population of vibrationally excited $\mathrm{NO}(\nu=1,2)$ is determined by a dynamic approach to equilibrium with the surface, controlled by rates of excitation given by (6), leading to a system of linear rate equations:

$$
\frac{\mathrm{d} n_{\nu^{\prime}}\left(t, T_{\mathrm{S}}\right)}{\mathrm{d} t}=\sum k_{\nu \nu^{\prime}} n_{\nu, \nu^{\prime}}\left(t, T_{\mathrm{S}}\right)
$$

The above-described approach to the analysis of the experimental results can be taken to different levels of complexity. Ultimately, one can allow both direct single and double $(\nu=0 \rightarrow 1$ and 2$)$ and two-step sequential $(\nu=0 \rightarrow 1 \rightarrow 2)$ excitation and relaxation processes to proceed concurrently, assembling a system of differential rate equations like shown 
in (7) that combines all possible excitation and relaxation pathways:

$$
\begin{aligned}
\frac{\mathrm{d} n_{0}}{\mathrm{~d} t} & =k_{10} n_{1}-k_{01} n_{0}-k_{02} n_{0}+k_{20} n_{2} \\
\frac{\mathrm{d} n_{1}}{\mathrm{~d} t} & =k_{01} n_{0}-k_{10} n_{1}-k_{12} n_{1}+k_{21} n_{2} \\
\frac{\mathrm{d} n_{2}}{\mathrm{~d} t} & =k_{12} n_{1}-k_{21} n_{2}+k_{02} n_{0}-k_{20} n_{2}
\end{aligned}
$$

Following our insight that sequential single quantum excitation is relatively unimportant, we first inspect these equations for the case neglecting $k_{1,2}$ and by detailed balance $k_{2,1}$. Solving the differential equations for this simplified single-step scenario yields the expression for absolute excitation probability, $P_{\nu \nu^{\prime}}$, as a function of the interaction time with the surface, $t$, and the surface temperature, $T_{\mathrm{S}}$ :

$$
P_{\nu \nu^{\prime}}=\frac{n_{\nu^{\prime}}(t)}{n_{0}(t=0)}=\frac{1-\exp \left[-a_{\nu \nu^{\prime}} E_{\nu \nu^{\prime}} \operatorname{coth}\left(\frac{E_{\nu \nu^{\prime}}}{2 k_{\mathrm{b}} T_{\mathrm{S}}}\right) t\right]}{1+\exp \left(\frac{E_{\nu \nu^{\prime}}}{k_{\mathrm{b}} T_{\mathrm{S}}}\right)}
$$

It can be shown ${ }^{12}$ that for $k_{\mathrm{b}} T_{\mathrm{S}} \ll E_{\nu \nu}^{\prime}$ (which is valid for the experiments of Fig. 9) and short interaction times $\left(t \ll 1 / k_{\nu \nu}^{\prime}\right)$, expression (9) can be reduced to:

$$
P_{\nu \nu^{\prime}} \approx k_{\nu \nu^{\prime}} t=\left(\alpha_{\nu \nu^{\prime}} E_{\nu \nu^{\prime}} t\right) \exp \left(-\frac{E_{\nu \nu^{\prime}}}{k_{\mathrm{b}} T_{\mathrm{S}}}\right)
$$

In expression (10), one recognizes the familiar Arrhenius form, which emerges as a low temperature and short interaction time limit of the more general expression given by eqn (9). The short interaction time limit criterion is shown in ref. 12 to be rather forgiving, leading to experimentally imperceptible deviations from Arrhenius behavior even for $k_{\nu \nu}^{\prime} t$ in the vicinity of half the thermal limit. Within this formalism it is clear how the empirically derived pre-exponential factor is related to the vibrational energy spacing, $E_{\nu \nu^{\prime}}$, the interaction time, $t$, and the fundamental coupling coefficient, $\alpha_{\nu \nu^{\prime}}$. This provides a link between experimentally derived quantities, $A_{\text {exp }, \nu}$ and intrinsic properties related to the electronic occupation of the LUMO and vibrational degree of freedom, $\lambda_{\nu \nu^{\prime}}$, and the density of electronic states in the metal projected onto the molecular affinity level, $\rho$. Thus experimentally measured quantities may be used to derive fundamental parameters of the Newns-Anderson Hamiltonian, assumed to be a good description of the electronically nonadiabatic interaction.

We also note that in the low $T_{\mathrm{S}}$ limit, where $k_{\mathrm{b}} T_{\mathrm{S}} \ll E_{\nu \nu}^{\prime}$, expression (9) obtains the form:

$$
P_{\nu \nu^{\prime}}=\exp \left(-\frac{E_{\nu \nu^{\prime}}}{k_{\mathrm{b}} T_{\mathrm{S}}}\right)
$$

in the limit of infinite interaction time. That is, in the limit of infinite molecule-surface interaction time the pre-exponential coefficient is unity, and the ratio between the amounts of molecules in two arbitrary vibrational states $\nu_{1}$ (with the energy $E_{\nu_{1}}$ ) and $\nu_{2}$ (with the energy $E_{\nu_{2}}$ ) is simply given by $\frac{n_{\nu_{2}}}{n_{\nu_{1}}}=\exp \left[-\frac{\left(E_{\nu_{2}}-E_{\nu_{1}}\right)}{k_{\mathrm{b}} T_{\mathrm{S}}}\right]$, as expected from thermal equilibrium. ${ }^{4,12}$

It is informative to carry out a similar analysis assuming the dominance of sequential single-quantum excitation, that is neglecting $k_{02}$ and $k_{20}$ in eqn (8). In the low $T_{\mathrm{S}}$ limit, $k_{\mathrm{b}} T_{\mathrm{S}} \ll E_{\nu \nu}^{\prime}$, and the short interaction time regime, one obtains:

$$
P_{\text {sequential }}(0-2)=\frac{1}{2}\left(\alpha_{01} E_{01} t\right)^{2} \exp \left(-\frac{2 E_{01}}{k_{\mathrm{b}} T_{\mathrm{S}}}\right)
$$

For the case of direct overtone $\mathrm{NO}(\nu=0 \rightarrow 2)$ excitation, it follows from eqn (10) that the absolute excitation probability is given by:

$$
P_{\text {direct }}(0-2)=\left(2 \alpha_{02} E_{01} t\right) \exp \left(-\frac{2 E_{01}}{k_{\mathrm{b}} T_{\mathrm{S}}}\right)
$$

assuming further the vibrational anharmonicity of the molecule is negligible. As we can see from expressions (12) and (13), the experimentally observed Arrhenius slope alone cannot be used to distinguish between the direct overtone and the sequential two-step mechanisms. On the other hand, the pre-exponential coefficients will differ significantly between the two excitation scenarios (compare eqn (12) and (13)) and can be used to reveal the branching ratio between the two.

Having obtained an intuitive understanding of the kinetic behavior using the simplified kinetic analysis described above, we can analyze the experimental data using the full kinetic model as reflected in the set of rate expressions given in eqn (8). All rate constants in eqn (8) are given by expression (6). Assuming (1) $\alpha_{\nu \nu}^{\prime}=\alpha_{\nu}^{\prime} \nu$ (detailed balance) and (2) $\alpha_{01}=\alpha_{12}$ (independence of the rates on initial vibrational state) we may define $\alpha_{02}=\beta \alpha_{01}$, where $\beta$ is the branching ratio between single step $\nu=0 \rightarrow 1$ and direct overtone $\nu=0 \rightarrow 2$ excitation. We also define the effective interaction time as $\tau=\alpha_{01} t$, to solve the system of rate equations (8).

The solutions of eqn (8) are functions $n_{0}\left(\tau, T_{\mathrm{S}}, \beta\right), n_{1}\left(\tau, T_{\mathrm{S}}, \beta\right)$, $n_{2}\left(\tau, T_{\mathrm{S}}, \beta\right)$ that can be used to obtain the corresponding excitation probabilities $P_{1}=\frac{n_{1}\left(\tau, T_{\mathrm{S}}, \beta\right)}{n_{0}\left(\tau=0, T_{\mathrm{S}}, \beta\right)}$ and $P_{2}=\frac{n_{2}\left(\tau, T_{\mathrm{S}}, \beta\right)}{n_{0}\left(\tau=0, T_{\mathrm{S}}, \beta\right)}$. Then, one can perform a global least squares fit on two experimental data sets $(\mathrm{NO}(\nu=1)$ and $\mathrm{NO}(\nu=2))$ simultaneously using the same pair of $\tau$ and $\beta$ as the adjustable parameters. Applying this analysis to the experimental data sets shown in Fig. 9 yields $\tau_{\text {eff }}=1.94[1 / \mathrm{eV}]$ and $\beta=0.68 .^{12}$ This global fitting procedure is rather robust, since the value of $\tau_{\text {eff }}$ is almost exclusively determined by the $\operatorname{NO}(\nu=1)$ data set, while the value of $\beta$ is mainly sensitive to the values of the $\mathrm{NO}(\nu=2)$ data set. ${ }^{4,12}$ This kinetic analysis allows one to factor out the influence of availability of suitably energetic EHPs, and to extract the intrinsic propensity for direct-overtone vibrational excitation in electronically non-adiabatic moleculemetal collision. Remarkably, this propensity turns out to be nearly as large as that for a single-quantum $(\nu=0 \rightarrow 1)$ excitation process $(\beta=0.68)$. The fits to the experimental data obtained from the full kinetic model are shown as solid lines in Fig. 9. The fact that this analysis is virtually indistinguishable from the canonical Arrhenius fits tells us that the experiments are in the low-temperature, short interaction time regime. . $^{42}$

Using the value of the effective interaction time, $\tau_{\text {eff }}$, obtained from fitting the solutions of the appropriate rate equations to the experimental data sets together with the independent estimate of the interaction time, it is possible to calculate the numerical values of the coefficients $\alpha_{\nu \nu^{\prime}}$. In recent work, focusing on exo-electrons emission occurring during the 
scattering of highly vibrationally excited NO from Cs-decorated $\mathrm{Au}$ surface ${ }^{47}$ a critical distance for vibrationally excited electron emission was identified as $Z_{\mathrm{C}} \approx 5 \AA$. Despite several important differences, this value can be taken as a characteristic of the interaction distance for vibrational excitation at least for the purpose of estimation. From the measured velocity of the NO molecular beam, we can estimate the turnaround time over twice the critical distance to be $\sim 400 \mathrm{fs}$. Using this number we can evaluate $\alpha_{01}=4.85 \times 10^{12}$ and $\alpha_{02}=3.16 \times 10^{12}(\mathrm{eV} \mathrm{s})^{-1}$. Although additional theoretical work is required to draw conclusions about the magnitude of these numbers, the very fact of their accessibility within the kinetic analysis employing the expression for vibrational energy transfer rate (eqn (6)) is useful.

The knowledge gained about the Arrhenius pre-exponential coefficients can be used to quantify the branching ratio between the direct overtone and sequential mechanism of $\mathrm{NO}(\nu=2)$ production in the course of $\mathrm{NO}(\nu=0) / \mathrm{Au}(111)$ collision. Assuming fractions of $\mathrm{NO}(\nu=2)$ produced by direct overtone and sequential mechanisms are $x$ and $y$, respectively, such that $x+y=1$, and assuming a short interaction time regime, the absolute excitation probability for $\operatorname{NO}(\nu=2)$ is given by the sum:

$$
P_{02}=\left[x \alpha_{02} t 2 E_{01}+\frac{y}{2}\left(\alpha_{01} t E_{01}\right)^{2}\right] \exp \left(-\frac{2 E_{01}}{k_{\mathrm{b}} T_{\mathrm{S}}}\right)
$$

The experimentally derived pre-exponential coefficients (see Fig. 9), $A_{\text {exp }, 2}$ and $A_{\text {exp }, 1}$, can be expressed as:

$$
\begin{aligned}
& A_{02}=x \alpha_{02} t 2 E_{01}+\frac{y}{2}\left(\alpha_{01} t E_{01}\right)^{2}=0.46 \\
& A_{01}=\alpha_{01} t E_{01}=0.38
\end{aligned}
$$

Using $\frac{\alpha_{02}}{\alpha_{01}}=\beta=0.68$ (as derived from the fit to the experimental data sets) and solving (15) together with $x+y=1$, we obtain that the branching ratio of direct overtone to sequential contributions is $0.86: 0.14$, respectively, demonstrating the dominance of the direct overtone process over the sequential mechanism of $\mathrm{NO}(\nu=2)$ vibrational excitation. This result also suggests that the vibrational relaxation of highly vibrationally excited NO exhibiting deposition of multiple vibrational quanta into excitation of a single $\mathrm{EHP}^{5,11,46,47}$ is mechanistically similar to the vibrational excitation of NO.

In this section we have shown how one can quantitatively evaluate electronically nonadiabatic interactions based on Fermi's Golden Rule. We return now to the question of the intrinsic coupling strengths in effect for $\mathrm{NO}(\nu=0)$ vibrational excitation and $\mathrm{NO}(\nu=15)$ vibrational relaxation. In terms of the thermal limit, we can see that the vibrational excitation of NO $(\nu=0)$ under the conditions of Fig. 9 reaches about half the thermal limit. By comparison the vibrational relaxation of $\mathrm{NO}(\nu=15)$ shown in Fig. 4a has gone from an initial value of $(\nu=15)$ to a final average value of the vibrational quantum number $\langle\nu\rangle \approx 8$. At thermal equilibrium, $\langle\nu\rangle=0$ as $T_{\mathrm{S}}$ was $300 \mathrm{~K}$. Thus we may also conclude that the vibrational relaxation of $\mathrm{NO}(\nu=15)$ is about half way to thermal equilibrium. This argument could be made more quantitative by extending the ideas of the state to state kinetic model, work that is left for the future. Nevertheless, one can see that the thermal limit represents a benchmark for evaluating the strength of the electronically nonadiabatic coupling and moreover, there is no major difference in the coupling strengths of $\operatorname{NO}(\nu=0)$ and for $\operatorname{NO}(\nu \gg 0)$.

\section{Applying the quantitative approach to compare different molecule-surface systems}

While EHP-V coupling is now a well-established mechanism of energy transfer in, for example, $\mathrm{NO} / \mathrm{Ag}, \mathrm{NO} / \mathrm{Cu}$ and $\mathrm{NO} / \mathrm{Au},{ }^{5,6,10,16,46,57,58}$ expanding the available range of observations to a wider variety of molecule/surface systems accompanied by connection to first principles theory is crucial to our ability to quantitatively predict the magnitude of electronically non-adiabatic interactions. An important step in this direction is the recent comprehensive set of measurements for vibrational excitation of $\mathrm{HCl}$ scattering from $\mathrm{Au}(111),{ }^{2,59}$ which, as opposed to the $\mathrm{NO} / \mathrm{Au}(111)$ system, appears to be a case of weak electronically non-adiabatic coupling. In these measurements - see Fig. 10 - the absolute excitation probability for $\operatorname{HCl}(\nu=0 \rightarrow 1)$ was studied as a function of $T_{\mathrm{S}}$ and $E_{\mathrm{I}}$. The absolute probabilities for $\nu=0 \rightarrow 1$ vibrational excitation of $\mathrm{HCl}\left(10^{-6}-10^{-4}\right)$ are markedly smaller than those of NO. They are sufficiently small that mechanical (electronically adiabatic) energy transfer can compete to some degree. ${ }^{2}$ Consequently, the $T_{\mathrm{S}}$ dependence is not simply Arrhenius. Rather, it exhibited a combination of Arrhenius, associated with an electronically nonadiabatic mechanism and temperature independent behavior associated with a mechanical mechanism:

$$
\begin{aligned}
P_{01}= & P_{01}^{\mathrm{mech}}\left(E_{l}\right)+P_{01}^{\mathrm{EHP}-\mathrm{V}}\left(E_{l}\right)=A_{01}^{\mathrm{mech}}\left(E_{l}\right) \\
& +A_{01}^{\mathrm{EHP}-\mathrm{V}}\left(E_{l}\right) \exp \left(-\frac{E_{01}}{k_{\mathrm{b}} T_{\mathrm{S}}}\right)
\end{aligned}
$$

In eqn (16), $A_{01}^{\text {mech }}\left(E_{l}\right)$ is the mechanical excitation probability assuming $T_{\mathrm{S}}$ independence. ${ }^{15}$ The second term of eqn (16)

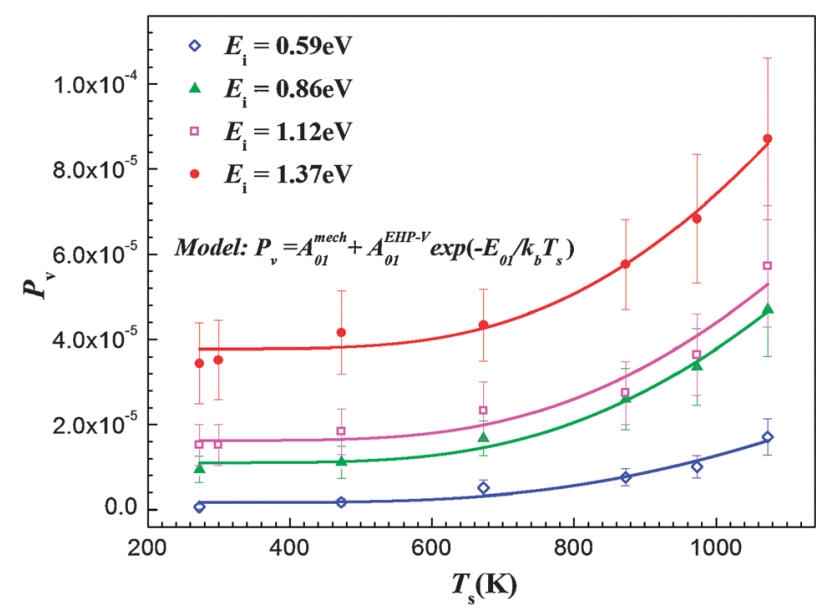

Fig. 10 Absolute vibrational excitation probabilities for $\mathrm{HCl}(\nu=0 \rightarrow 1)$ scattering from $\mathrm{Au}(111) \mathrm{vs}$. $T_{\mathrm{S}}$ measured at several values of $E_{\mathrm{I}}$. The symbols are experimental measurements and the solid lines are fits describing two contributions to the scattering: electronically adiabatic and nonadiabatic. See the text and especially eqn (17). Adapted from ref. 2. 
represents electronically nonadiabatic excitation where the activation energy is the $\operatorname{HCl}(\nu=0-1)$ vibrational spacing $(0.371 \mathrm{eV})$. The $A_{01}^{\mathrm{EHP}-\mathrm{V}}\left(E_{l}\right)$ factor is the Arrhenius pre-exponent associated with the inherent strength of EHP-V coupling. ${ }^{4,12}$ Eqn (17) was used to fit the data of Fig. 10, leading to values for $A_{01}^{\text {mech }}\left(E_{l}\right)$ and $A_{01}^{\mathrm{EHP}-\mathrm{V}}\left(E_{l}\right)$. This enabled estimation of the relative importance of these competing excitation channels. ${ }^{2}$ In this way the two contributing components (adiabatic and nonadiabatic) could be disentangled from one another. The dependence of the adiabatic component on $E_{\mathrm{I}}$ exhibited a marked threshold at $E_{\mathrm{I}} \approx 0.57 \mathrm{eV}$, indicating conversion of incident translational energy to vibration, rotation and surface excitation. ${ }^{60,61}$ This channel was also $T_{\mathrm{S}}$ independent similar to $\mathrm{NH}_{3}$ on $\mathrm{Au}(111)$ shown in Fig. 1c. ${ }^{2}$

The electronically nonadiabatic component showed characteristic threshold-less $E_{\mathrm{I}}$ dependence and Arrhenius $T_{\mathrm{S}}$ dependence. ${ }^{2}$ These results are shown more clearly in Fig. 11 as green lines and symbols. At low values of $T_{\mathrm{S}}$, the adiabatic contribution dominates. With increasing $T_{\mathrm{S}}$, the contribution of the electronically nonadiabatic channel rapidly increases. Fig. 11 also shows the previously discussed $\operatorname{NO}(\nu=0 \rightarrow 1,2)$ excitation probabilities $^{4,12}$ along with earlier measurements of $\mathrm{NO}(\nu=0 \rightarrow 1)$ excitation upon collision with $\mathrm{Cu}(110)^{16}$ and $\mathrm{Ag}(111) .^{6,25}$ All NO/metal systems, without exception, show substantially higher excitation probabilities than those of $\mathrm{HCl}$ ( $\sim 2-4$ orders of magnitude). Even the excitation probabilities for multi-quantum $\mathrm{NO}(\nu=0 \rightarrow 2)$ excitation, which is being reduced by "unfavorable" activation energy of $0.472 \mathrm{eV}$ (two vibrational spacings of NO), are higher than these of

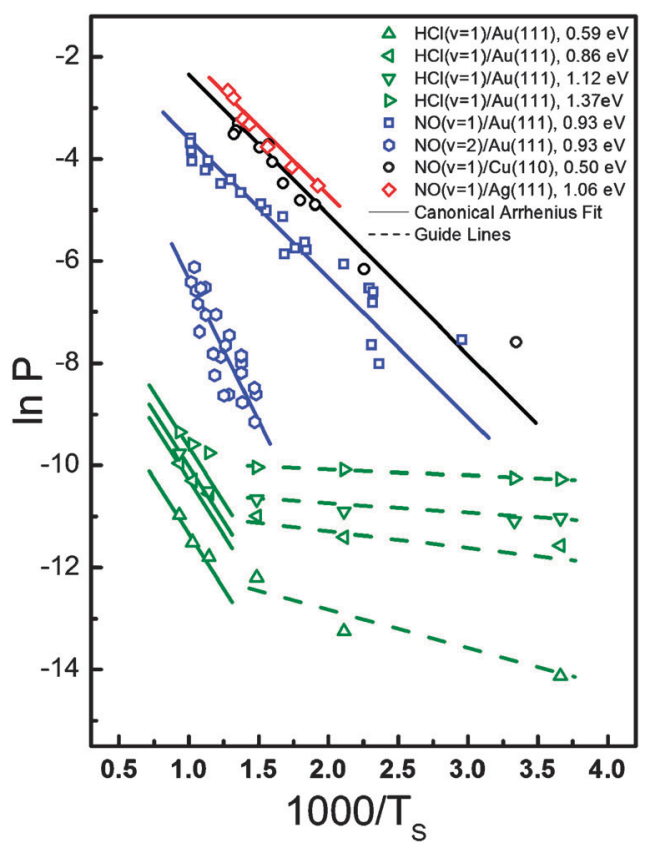

Fig. 11 Arrhenius plots for vibrational excitation probabilities of: $\mathrm{HCl}(v=0 \rightarrow 1) / \mathrm{Au}(111)$ at four different $E_{\mathrm{I}}{ }^{\prime} \mathrm{s}^{2} ; \mathrm{NO}(v=0 \rightarrow 1,2) /$ $\mathrm{Au}(111) ;{ }^{4,12} \mathrm{NO}(v=0 \rightarrow 1) / \mathrm{Cu}(110) ;{ }^{16} \mathrm{NO}(v=0 \rightarrow 1) / \operatorname{Ag}(111) .{ }^{6}$ The solid lines represent the canonical Arrhenius fit with an activation energy fixed to the value of the corresponding vibrational excitation; the dashed lines are drawn to guide the eye where the vibrational excitation of $\mathrm{HCl}$ is dominated by an adiabatic mechanism.
$\operatorname{HCl}(\nu=0 \rightarrow 1)$, having more "favorable" activation energy of $0.371 \mathrm{eV}$ (one vibrational spacing of $\mathrm{HCl}$ ). It is clear that the inherent propensity for vibrational excitation is significantly higher for $\mathrm{NO}$ than for $\mathrm{HCl}$.

We now show by example how the electronically adiabatic coupling strength can be compared between two systems. Consider $\mathrm{NO}$ and $\mathrm{HCl}$ data obtained at a similar $E_{\mathrm{I}}$. Based on a similar analysis as that just presented, $\alpha_{01}$ to be $\sim 1.17 \times 10^{10}(\mathrm{eV} \mathrm{s})^{-1}$ is obtained for $\mathrm{HCl}$ scattering from $\mathrm{Au}$ at $E_{\mathrm{I}}=0.86 \mathrm{eV}$. Recall that for $\mathrm{NO}$ molecules with an incidence kinetic energy of $0.93 \mathrm{eV}$ scattering from $\mathrm{Au}(111)$, the EHP-V coupling strength parameters were found to be $\alpha_{01}=4.85 \times 10^{12}(\mathrm{eV} \mathrm{s})^{-1}$ for single-quantum excitation. ${ }^{12}$ Hence there is a $\sim 400$-fold difference in the EHP-V coupling strength between $\mathrm{HCl}$ and $\mathrm{NO}$ on $\mathrm{Au}(111)$. Alternatively one can estimate the fraction of the thermal limit that is seen in vibrational excitation, which is relatively independent of $T_{\mathrm{S}}$. One finds that for NO, the vibrational populations seen from $\mathrm{Au}(111)$ scattering at $E_{\mathrm{I}}=0.93 \mathrm{eV}$ are about 0.4 of the thermal limit, whereas for $\mathrm{HCl}$ they are about 0.002 , about a 200 -fold difference. To compare $\alpha_{01}$ to populations we should correct for the vibrational excitation energy and interaction time difference between to the two systems - see eqn (10). This leads to the conclusion that $\mathrm{HCl}$ is $300-400$ times further from the thermal limit than $\mathrm{NO}$ in collision with $\mathrm{Au}(111)$ at $E_{\mathrm{I}} \approx 0.9 \mathrm{eV}$. The uncertainty derives from the differential interaction time. While the state to state model leads to more rigorous results and numerical values for $\alpha_{\nu \nu^{\prime}}$, even the simple estimate using the thermal limit is highly informative.

We may also compare to prior experiments. Data are available for electronically nonadiabatic vibrational excitation of $\operatorname{NO}(\nu=0 \rightarrow 1)$ upon collision with $\operatorname{Ag}(111)^{6,25}$ and $\mathrm{Cu}(110) .{ }^{16}$ Here we are led to the conclusion that there are experimental problems with the reported data as the pre-exponent extracted from canonical Arrhenius analysis exceeds unity$A_{\text {exp }, 1}=2.0$ for $\operatorname{Ag}(111)$ and $A_{\text {exp }, 1}=1.3$ for $\mathrm{Cu}(110)$. According to the analysis presented here, the pre-exponent cannot exceed unity. Clearly both these scattering systems are close to the thermal limit. Measuring absolute excitation probabilities is difficult and we believe that additional experimental effort is required to resolve this discrepancy.

The comparison of $\mathrm{NO}$ and $\mathrm{HCl}$ on $\mathrm{Au}(111)$ gives an idea how different in magnitude the electronically non-adiabatic interactions can be. Obviously, development of predictive first principles theory is the ultimate goal. Recent progress using independent electron surface hopping represents progress in this direction. ${ }^{58,62,63}$

Until this and related theories become better proven we seek "intuitive proxies" that might help guide our understanding. The energy of the affinity level is one such proxy. According to the model proposed by Newns, ${ }^{10}$ the energy of the molecule's affinity level at the distance of closest approach to the metal surface during the scattering process strongly influences the electronically non-adiabatic coupling. In the limit of infinite separation from the metal, the energy affinity level is simply the difference of the surface work function and molecule's electron affinity. As the molecule approaches the surface, the affinity level is strongly stabilized by image-charge interaction. If we assume that the distance of closest approach is identical 


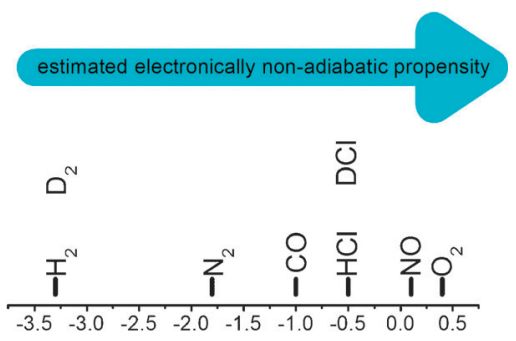

asymptotic electron affinity, $\mathrm{eV}$

Fig. 12 Series of diatomics ordered according to their asymptotic electron affinity. The blue arrow shows a tentative trend attempting to predict the electronically non-adiabatic propensity of these diatomics.

(or at least similar) for different molecules, then for a single metal, the higher the molecule's electron affinity, the lower the affinity level will be at the closest approach, and thus the stronger will be the expected nonadiabatic coupling. One might imagine using this proxy to at least order from weakest to strongest, the strength of the electronically nonadiabatic interactions. Fig. 12 shows a series of diatomics ordered according to their electron affinities. Several of the molecules listed in Fig. 12 have negative electron affinities, indicating that the anion is higher in energy than the neutral. The negative electron affinity can nevertheless be determined from negative ion resonance data. ${ }^{64-68}$ Clearly when considering a molecule interacting with different metals, the work function would be an analogous proxy. One would expect low work function surfaces to have much stronger electronically nonadiabatic interactions than high work function surfaces. There is as yet no systematic data to show this effect, although it is worth pointing out that the ordering of $\mathrm{NO}$ on $\mathrm{Au}, \mathrm{Ag}$ and $\mathrm{Cu}$ is in accord with these expectations based on published work functions. ${ }^{69}$

Additional factors could of course be considered in a rational attempt to order the strengths of nonadiabatic interactions; for example, negative ion resonance widths in the vicinity of the metal surface. Unfortunately, such data are hard to find. We hope that these ideas will stimulate future work to help establish trends in nonadiabatic coupling strengths.

\section{Prospects for future development of the quantitative approach}

The quantitative approach presented here assumes that the interaction rate, $k_{\nu \nu^{\prime}}$ (or equivalently, $\alpha_{\nu v^{\prime}}$ ) is constant during the molecule-surface collision. A more realistic description would account for the fact that the affinity level approaches the Fermi level due to Coulomb image charge stabilization allowing formation of a short-lived anionic state. The rapid return of the electron to the metal results in lifetime broadening of the molecular affinity level, which decreases with the distance from the surface as indicated in Fig. 13. The next step in improving the quantitative approach incorporates the molecule-surface distance dependence of $\alpha_{\nu \nu^{\prime}}$.

We next outline what is necessary for this to be implemented. By modifying the rate equations (8), using time dependent rate

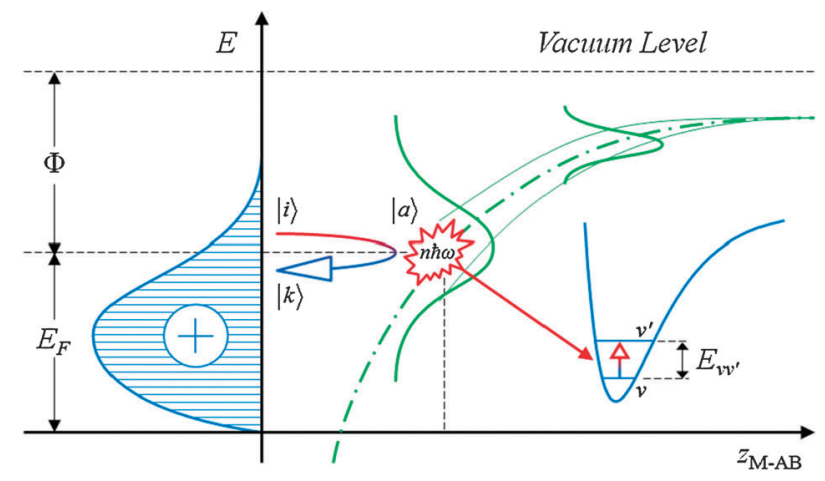

Fig. 13 Energy vs. the molecule surface distance. Hatched area depicts conduction electrons (with positive image charge) as the product of the Fermi-Dirac function (with the Fermi level $E_{\mathrm{F}}$ ) and the 3D density of states; $\Phi$ is the work function of the metal surface; $|a\rangle$ is the affinity level experiencing lifetime broadening and image charge stabilization; $|i\rangle$ and $|k\rangle$ are the initial and final electronic states in the metal.

"constants", we obtain a similar mathematical problem to solve as we have discussed above, e.g.:

$$
\begin{aligned}
\frac{\mathrm{d} n_{0}}{\mathrm{~d} t} & =k_{10}(t) n_{1}-k_{01}(t) n_{0}-k_{02}(t) n_{0}+k_{20}(t) n_{2} \\
\frac{\mathrm{d} n_{1}}{\mathrm{~d} t} & =k_{01}(t) n_{0}-k_{10}(t) n_{1}-k_{12}(t) n_{1}+k_{21}(t) n_{2} \\
\frac{\mathrm{d} n_{2}}{\mathrm{~d} t} & =k_{12}(t) n_{1}-k_{21}(t) n_{2}+k_{02}(t) n_{0}-k_{20}(t) n_{2}
\end{aligned}
$$

which can be further parameterized with the molecule-surface distance $z$.

$$
k_{\nu \nu^{\prime}}(z)=\frac{\alpha_{\nu \nu^{\prime}}(z) E_{\nu \nu^{\prime}}}{\exp \left(\frac{E_{\nu \nu^{\prime}}}{k_{\mathrm{b}} T_{\mathrm{S}}}\right)-1} \quad \text { with } \quad \alpha_{\nu \nu^{\prime}}=\frac{2 \pi}{\hbar} \lambda_{\nu \nu^{\prime}}^{2} \rho(z)^{2}
$$

Here, the time dependence is recovered from $z=v t$ and $\mathrm{d} z=v \mathrm{~d} t$, with the molecular velocity inferred from the incidence kinetic energy, $E_{\mathrm{I}}$ and the interaction potential, $U(z)$ :

$$
\nu(z)=\sqrt{\frac{2}{m}\left[E_{l}-U(z)\right]}
$$

The interaction potential may be taken as a realistically parameterized Lennard-Jones potential that could be derived from quantum chemical calculations of the $\mathrm{NO} / \mathrm{Au}$ interaction potential. ${ }^{62}$ One could then extract fundamental quantities from the Newns-Anderson Hamiltonian from systematic measurements of absolute excitation/de-excitation probabilities at different $T_{\mathrm{S}}$ and $E_{\mathrm{I}}$. Such data are now available for $\mathrm{NO}$ on $\mathrm{Au}(111)$ at 6 values of $E_{\mathrm{I}}$ between 0.1 and $1.2 \mathrm{eV}$ and at several values of $T_{\mathrm{S}}$ between 300 and $1100 \mathrm{~K}$. Preliminary results, extending the work highlighted in this paper, accurately reproduce the $E_{\mathrm{I}}$ and $T_{\mathrm{S}}$ dependence of experiment and will be presented in a future publication.

\section{Acknowledgements}

The authors gratefully acknowledge financial support from the Department of Energy Office of Basic Energy Sciences, under Grant No. DE-FG02-03ER15441 as well as the Partnership 
for International Research and Education-for Electronic Chemistry and Catalysis at Interfaces-NSF Grant No. OISE-0530268. AMW would also like to acknowledge the Max Planck Institute for Biophysical Chemistry and Institute for Physical Chemistry at the Georg-August University of Göttingen. IR gratefully acknowledges The Open University of Israel Research Authority Grant No. 47324.

\section{References}

1 Y. J. Chabal, Electronic damping of hydrogen vibration on the W (100) surface, Phys. Rev. Lett., 1985, 55(8), 845-848.

2 Q. Ran, et al., Observation of a change of vibrational excitation mechanism with surface temperature: $\mathrm{HCl}$ collisions with $\mathrm{Au}(111)$, Phys. Rev. Lett., 2007, 98(23), 237601.

3 M. Morin, N. J. Levinos and A. L. Harris, Vibrational-energy transfer of $\mathrm{CO} / \mathrm{Cu}(100)$-nonadiabatic vibration electron coupling, J. Chem. Phys., 1992, 96(5), 3950-3956.

4 R. Cooper, et al., Vibrational overtone excitation in electron mediated energy transfer at metal surfaces, Chem. Sci., 2010, 1(1), 55-61.

5 Y. H. Huang, et al., Vibrational promotion of electron transfer, Science, 2000, 290(5489), 111-114.

6 C. T. Rettner, et al., Observation of direct vibrational-excitation in gas-surface collisions-NO on $\mathrm{Ag}(111)$, Phys. Rev. Lett., 1985, 55(18), 1904-1907.

7 J. L. Larue, et al., The work function of submonolayer cesium-covered gold: A photoelectron spectroscopy study, J. Chem. Phys., 2008, 129(2), 024709.

8 D. C. Langreth, Energy-transfer at surfaces-asymmetric line-shapes and the electron-hole pair mechanism, Phys. Rev. Lett., 1985, 54(2), 126-129.

9 H.-C. Chang and G. E. Ewing, Infrared fluorescence from a monolayer of $\mathrm{CO}$ on $\mathrm{NaCl}(100)$, Phys. Rev. Lett., 1990, 65(17), 2125 .

10 D. M. Newns, Electron-hole pair mechanism for excitation of intramolecular vibrations in molecule surface scattering, Surf. Sci., 1986, 171(3), 600-614.

11 J. LaRue, et al., Vibrationally promoted electron emission at a metal surface: electron kinetic energy distributions, Phys. Chem. Chem. Phys., 2011, 13, 97-99.

12 D. Matsiev, et al., On the temperature dependence of electronically non-adiabatic vibrational energy transfer in molecule-surface collisions, Phys. Chem. Chem. Phys., 2011, 13, 8153-8162.

13 A. M. Wodtke, D. Matsiev and D. J. Auerbach, Energy transfer and chemical dynamics at solid surfaces: The special role of charge transfer, Prog. Surf. Sci., 2008, 83(3), 167-214.

14 M. Born and J. R. Oppenheimer, On the quantum theory of molecules, Ann. Phys., 1927, 84, 457-484.

15 B. D. Kay, T. D. Raymond and M. E. Coltrin, Observation of direct multiquantum vibrational-excitation in gas-surface scattering- $\mathrm{NH}_{3}$ on Au(111), Phys. Rev. Lett., 1987, 59(24), 2792-2794.

16 E. K. Watts, J. L. W. Siders and G. O. Sitz, Vibrational excitation of NO scattered from Cu(110), Surf. Sci., 1997, 374(1-3), 191-196.

17 J. C. Tully, Perspective on "Zur Quantentheorie der Molekeln": Born M, Oppenheimer R. (1927) Ann Phys 84:457, Theor. Chem. Acc., 2000, 103, 173-176.

18 A. M. Wodtke, J. C. Tully and D. J. Auerbach, Electronically non-adiabatic interactions of molecules at metal surfaces: Can we trust the Born-Oppenheimer approximation for surface chemistry?, Int. Rev. Phys. Chem., 2004, 23(4), 513-539.

19 M. Silva, et al., The dynamics of "stretched molecules": Experimental studies of highly vibrationally excited molecules with stimulated emission pumping, Аппи. Rev. Phys. Chem., 2001, 52, $811-852$.

20 A. W. Kleyn, A. C. Luntz and D. J. Auerbach, Rotational energytransfer in direct inelastic surface scattering- NO on $\operatorname{Ag}(111)$, Phys. Rev. Lett., 1981, 47(16), 1169-1172.

21 C. T. Rettner, et al., Chemical dynamics at the gas-surface interface, J. Phys. Chem., 1996, 100(31), 13021-13033.

22 G. O. Sitz, Gas surface interactions studied with state-prepared molecules, Rep. Prog. Phys., 2002, 65(8), 1165-1193.
23 K. C. Janda, et al., Direct measurement of velocity distributions in argon beam-tungsten surface scattering, J. Chem. Phys., 1980, 72(4), 2403-2410.

24 J. E. Hurst, et al., Direct inelastic-scattering $\operatorname{Ar}$ from $\operatorname{Pt}(111)$, J. Chem. Phys., 1983, 78(3), 1559-1581.

25 C. T. Rettner, et al., Direct vibrational-excitation in gas surfacecollisions of NO with $\operatorname{Ag}(111)$, Surf. Sci., 1987, 192(1), 107-130.

26 B. D. Kay, T. D. Raymond and M. E. Coltrin, Observation of a dynamical propensity rule in rotationally inelastic gas-surface

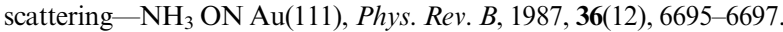

27 B. N. J. Persson and R. Ryberg, Vibrational damping of adsorbed molecules - methoxide on $\mathrm{Cu}(100)$, Phys. Rev. Lett., 1982, 48(8), 549-552.

28 B. N. J. Persson and R. Ryberg, Vibrational phase relaxation at surfaces - CO on Ni(111), Phys. Rev. Lett., 1985, 54(19), 2119-2122.

29 B. N. J. Persson and R. Ryberg, Brownian-motion and vibrational phase relaxation at surfaces-CO on Ni(111), Phys. Rev. B, 1985, 32(6), 3586-3596.

30 R. Ryberg, Carbon-monoxide adsorbed on $\mathrm{Cu}(100)$ studied by infrared-spectroscopy, Surf. Sci., 1982, 114(2-3), 627-641.

31 R. Ryberg, Vibrational line-shape of chemisorbed CO, Phys. Rev. $B, 1985,32(4), 2671-2673$.

32 R. Ryberg, The oxidation of methanol on $\mathrm{Cu}(100)$, J. Chem. Phys., $1985,82(1), 567-573$.

33 S. Chiang, R. G. Tobin and P. L. Richards, Vibrational spectroscopy of chemisorbed molecules by infrared-emission, J. Vac. Sci. Technol., A, 1984, 2(2), 1069-1074.

34 S. Chiang, et al., Molecule-substrate vibration of $\mathrm{CO}$ on $\mathrm{Ni}(100)$ studied by infrared-emission spectroscopy, Phys. Rev. Lett., 1984, 52(8), 648-651.

35 R. G. Tobin and P. L. Richards, An infrared-emission study of the molecule substrate mode of CO on Pt(111), Surf. Sci., 1987, 179(2-3), 387-403.

36 J. C. Ariyasu, et al., Anharmonic damping of adsorbate vibrationalmodes, Phys. Rev. B: Condens. Matter Mater. Phys., 1983, 28(10), 6123-6126.

37 E. J. Heilweil, et al., Picosecond vibrational-energy transfer studies of surface adsorbates, Annu. Rev. Phys. Chem., 1989, 40, 143-171.

38 E. J. Heilweil, R. R. Cavanagh and J. C. Stephenson, PIcosecond study of the population lifetime of $\mathrm{CO}(v=1)$ chemisorbed on $\mathrm{SiO}_{2}$-supported rhodium particles, J. Chem. Phys., 1988, 89(8), $5342-5343$.

39 J. D. Beckerle, et al., Subpicosecond transient infrared-spectroscopy of adsorbates-vibrational dynamics of $\mathrm{CO} / \mathrm{Pt}(111)$, J. Chem. Phys., 1991, 95(7), 5403-5418.

$40 \mathrm{~S}$. A. Corcelli and J. C. Tully, Vibrational energy pooling in $\mathrm{CO}$ on NaCl(100): Methods, J. Chem. Phys., 2002, 116(18), 8079-8092.

$41 \mathrm{~S}$. A. Corcelli and J. C. Tully, Vibrational energy pooling in CO on $\mathrm{NaCl}(100)$ : Simulation and isotope effect, J. Phys. Chem. A, 2002, 106(45), 10849-10860.

42 Y. Huang, et al., Observation of vibrational excitation and deexcitation for $\mathrm{NO}(\mathrm{v}=2)$ scattering from $\mathrm{Au}(111)$ : Evidence for electron-hole-pair mediated energy transfer, Phys. Rev. Lett., 2000, 84(13), 2985-2988.

43 J. D. White, et al., Vibrationally promoted electron emission from low work-function metal surfaces, J. Chem. Phys., 2006, 124(6), 064702.

44 N. H. Nahler and A. M. Wodtke, Dynamics of molecule-induced electron emission from surfaces, Mol. Phys., 2008, 106(16-18), $2227-2244$.

45 S. Monturet and P. Saalfrank, Role of electronic friction during the scattering of vibrationally excited nitric oxide molecules from Au(111), Phys. Rev. B: Condens. Matter Mater. Phys., 2010, 82(7), 075404.

46 J. D. White, et al., Conversion of large-amplitude vibration to electron excitation at a metal surface, Nature, 2005, 433(7025), 503-505.

47 N. H. Nahler, et al., Inverse velocity dependence of vibrationally promoted electron emission from a metal surface, Science, 2008, 321(5893), 1191-1194.

48 A. Bottcher, et al., Exoelectron emission at Cs surfaces by accelerated $\mathrm{O}_{2}$ molecules, Chem. Phys. Lett., 1994, 231(1), 119-122.

49 G. Ertl, Dynamics of reactions at surfaces, Adv. Catal., 2000, 45, $1-69$.

50 G. Ertl, Heterogeneous catalysis on atomic scale, J. Mol. Catal. A: Chem., 2002, 182(1), 5-16. 
51 G. Ertl and J. Küppers, Low energy electrons and surface chemistry, $\mathrm{VCH}, 1985$.

$52 \mathrm{~T}$. Greber, et al., On the dissociation of $\mathrm{O}_{2}$ on alkali metals, Surf. Rev. Lett., 1995, 2(3), 273-277.

$53 \mathrm{~W}$. Sesselmann, et al., Interaction of metastable noble-gas atoms with transition-metal surfaces: Resonance ionization and Auger neutralization, Phys. Rev. B: Condens. Matter Mater. Phys., 1987, 35(4), 1547.

54 B. N. J. Persson and M. Persson, Vibrational lifetime for CO adsorbed on $\mathrm{Cu}(100)$, Solid State Commun., 1980, 36(2), 175-179.

55 P. W. Anderson, Localized magnetic states in metals, Phys. Rev., 1961, 124(1), 41-53.

56 D. M. Newns, Fermi-Thomas response of a metal surface to an external point charge, J. Chem. Phys., 1969, 50(10), 4572-4575.

57 N. Shenvi, et al., Vibrational relaxation of $\mathrm{NO}$ on $\mathrm{Au}(111)$ via electron-hole pair generation, J. Chem. Phys., 2006, 125(15), 154703.

58 N. Shenvi, S. Roy and J. C. Tully, Dynamical steering and electronic excitation in NO scattering from a gold surface, Science, 2009, 326(5954), 829-832.

59 Q. Ran, et al., Direct translation-to-vibrational energy transfer of $\mathrm{HCl}$ on gold: Measurement of absolute vibrational excitation probabilities, Nucl. Instrum. Methods Phys. Res., Sect. B, 2007, 258(1), $1-6$.

60 I. Rahinov, et al., Efficient vibrational and translational excitations of a solid metal surface: State-to-state time-of-flight measurements of $\operatorname{HCl}(v=2, J=1)$ scattering from $\mathrm{Au}(111), J$. Chem. Phys., 2008, 129(21), 214708.

61 R. Cooper, et al., Efficient translational excitation of a solid metal surface: State-to-state translational energy distributions of vibrational ground state $\mathrm{HCl}$ scattering from $\mathrm{Au}$ (111), AVS, 2009.

62 S. Roy, N. A. Shenvi and J. C. Tully, Model Hamiltonian for the interaction of NO with the $\mathrm{Au}(111)$ surface, J. Chem. Phys., 2009, 130(17), 174716.

63 N. Shenvi, S. Roy and J. C. Tully, Nonadiabatic dynamics at metal surfaces: Independent-electron surface hopping, J. Chem. Phys., 2009, 130(17), 174107.

64 G. J. Schulz, Resonances in electron impact on diatomic molecules, Rev. Mod. Phys., 1973, 45, 423.

$65 \mathrm{~J}$. Horacek, et al., Isotope effects in vibrational excitation and dissociative electron attachment of $\mathrm{DCl}$ and DBr, Eur. Phys. J. D, 2005, 35(2), 225-230.

$66 \mathrm{M}$. Allan, et al., Electron scattering in cooled $\mathrm{HCl}$ : boomerang structures and outer-well resonances in elastic and vibrational excitation cross sections, J. Phys. B: At., Mol. Opt. Phys., 2000, 33(6), L209-L213.

67 J. N. Bardsley and J. M. Wadehra, Dissociative attachment in $\mathrm{HCl}, \mathrm{DCl}$ and $\mathrm{F}_{2}$, J. Chem. Phys., 1983, 78(12), 7227-7234.

$68 \mathrm{~K}$. Rohr and F. Linder, Vibrational-excitation in e- $\mathrm{HCl}$ collisions at low energies, J. Phys. B: At., Mol. Opt. Phys., 1975, 8(10), L200-L204.

69 B. J. Hopkins and J. C. Riviere, Work function of polycrystalline tungsten foil, Proc. Phys. Soc., London, 1963, 81(521), 590. 